\title{
Surface Mobility of Molecular Glasses and Its Importance in Physical Stability
}

\author{
Lian Yu \\ University of Wisconsin - Madison, School of Pharmacy and Department of Chemistry
}

\begin{abstract}
Amorphous molecular materials (molecular glasses) are useful for drug delivery, biopreservation and organic electronics. A central issue in developing amorphous materials is the stability against crystallization and other transformations that can compromise material performance. We review recent progress in understanding the stability of molecular glasses, particularly the role for surface mobility. Surface diffusion in molecular glasses can be vastly faster than bulk diffusion. This high surface mobility enables fast crystal growth on the free surface. In this process, surface crystals grow upward and laterally, with the lateral growth rate being roughly proportional to surface diffusivity. Surface mobility also influences bulk crystal growth as the process can create fracture and free surfaces. During vapor deposition, surface mobility allows efficient equilibration of newly deposited molecules, producing low-energy, high-density glasses that are equivalent to liquid-cooled glasses aged for thousands of years. Free surfaces can accelerate chemical degradation of proteins. Measures for inhibiting surface-facilitated transformations include minimizing free surfaces, applying surface coatings, and preventing fracture.
\end{abstract}




\section{INTRODUCTION}

Glasses are amorphous materials that combine the mechanical strength of crystals and the spatial uniformity of liquids. Compared to crystals, glasses are more easily fabricated to be spatially homogeneous from macroscopic to nearly molecular dimensions. Such spatial uniformity is the basis for their wide applications in optics and contributes to the superior strength of metallic glasses. ${ }^{1,2}$ While better-known glasses are inorganic and polymeric, organic glasses of relatively low molecular weights ("molecular glasses") are being explored for applications in drug delivery, ${ }^{3,4,5}$ bio-preservation,,${ }^{6,7}$ and organic electronics. ${ }^{8,9}$ Pharmaceutical scientists take advantage of the high solubility of amorphous solids for the delivery of poorly soluble drugs.

An important subject in the study of molecular glasses is physical stability. ${ }^{5,10,11}$ Glasses are nonequilibrium solids formed by cooling liquids, condensing vapors, or evaporating solutions while avoiding crystallization. A common feature of these processes is the kinetic arrest of a fluid structure. In glass formation by liquid cooling (Figure 1), molecular motions slow down with falling temperature and eventually the system falls out of equilibrium at the so-called glass transition temperature $T_{\mathrm{g}}$, forming a solid glass. Glasses are thermodynamically driven to crystallize and to "age" toward the equilibrium liquid state, both processes leading to changes in structure, properties, and performance.

A recent progress in understanding glass stability is the finding that molecular glasses have extremely high surface mobility and

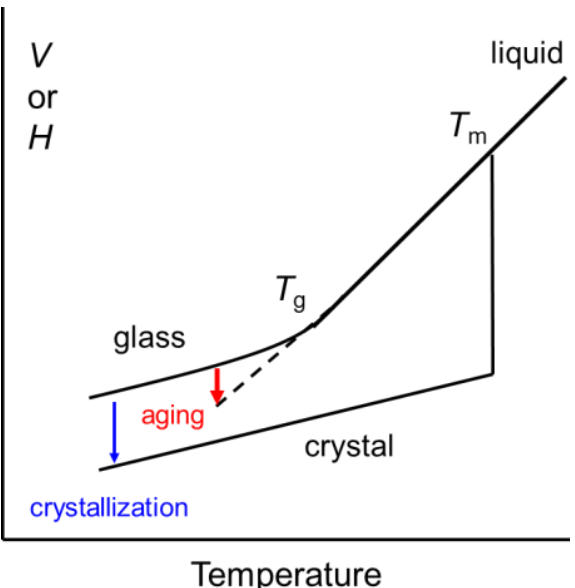

Figure 1. Formation, crystallization and aging of glasses. Cooling a liquid without crystallization produces a glass below $T_{\mathrm{g}}$ (the glass transition temperature). $V$ is volume; $H$ is enthalpy. A glass is driven to crystallize and age toward the supercooled liquid. this property causes problems of poor stability and paradoxically, provides a tool for preparing glasses with vastly improved stability. Here we discuss this recent progress. Section II reviews recent measurements of surface diffusion on molecular glasses. Section III discusses the role of surface 
mobility in the physical stability of molecular glasses. We show that surface mobility is directly responsible for fast crystal growth on free surfaces, and may be involved in bulk crystal growth through the creation of voids and free surfaces. In vapor deposition, surface mobility allows efficient equilibration of newly deposited molecules and the formation of stable glasses with exceptionally low energy and high density. In Section IV, we consider the methods for stabilizing molecular glasses against surface-facilitated transformations.

\section{SURFACE MOBILITY OF MOLECULAR GLASSES}

Recent experiments have shown that molecules on the free surface of an organic glass can be much more mobile than those in bulk. The experiments that led to this conclusion include the evolution of surface contours driven by surface tension, ${ }^{12,13,14,15,16}$ the conductivity of ions implanted at different depths,${ }^{17}$ the embedding of nano-particles, ${ }^{18}$ and the rotation of probe molecules. ${ }^{19}$ The high mobility of surface molecules is qualitatively understood from their special environment: a surface molecule has fewer neighbors than a bulk molecule and a greater freedom of movement.

Diffusion can be vastly faster on the free surface of a molecular glass than in the interior. ${ }^{12,13,14}$ Here, surface diffusion refers to the lateral translation of surface molecules. Figure 2 compares the coefficients of surface diffusion and bulk diffusion, $D_{\mathrm{s}}$ and $D_{\mathrm{v}}$, for several systems. The temperature is scaled by $T_{\mathrm{g}}$, which allows the $D_{\mathrm{v}}$ values of different systems to approximately collapse to one master curve, serving as a reference for comparing surface diffusivity. For the three systems in Figure 2, indomethacin (IMC), Nifedipine (NIF), and $o$-terphenyl (OTP), $D_{\mathrm{s}} / D_{\mathrm{v}}$ is $10^{6}$ to $10^{8}$ at $T_{\mathrm{g}}$ and the ratio increases with cooling, since $D_{\mathrm{v}}$ decreases faster than $D_{\mathrm{s}}$. The fast surface diffusion suggests the importance of surfacefacilitated processes, as we discuss later.

The results in Figure 2 indicate that surface diffusion slows down as intermolecular forces increase. At the same $D_{\mathrm{v}}$, surface diffusion follows the order OTP > NIF > IMC, or the opposite order for 
intermolecular forces. To see this, note that OTP is the smallest molecule and forms no hydrogen bonds. NIF and IMC are similar in size and IMC forms stronger hydrogen bonds (for its carboxyl group) than NIF. The physical picture is that the small, loosely bound OTP molecules are freer to diffuse on the surface than the larger, more strongly bound NIF and IMC molecules. In very recent work, this picture was extended to include low-molecular-weight polymers. ${ }^{20}$ The surface diffusion of polystyrene oligomers $\left(M_{\mathrm{w}}=1-2 \mathrm{~kg} / \mathrm{mol}\right)$ is significant slower than that of the small molecules, with $D_{\mathrm{s}} / D_{\mathrm{v}} \approx 10^{5}$ at
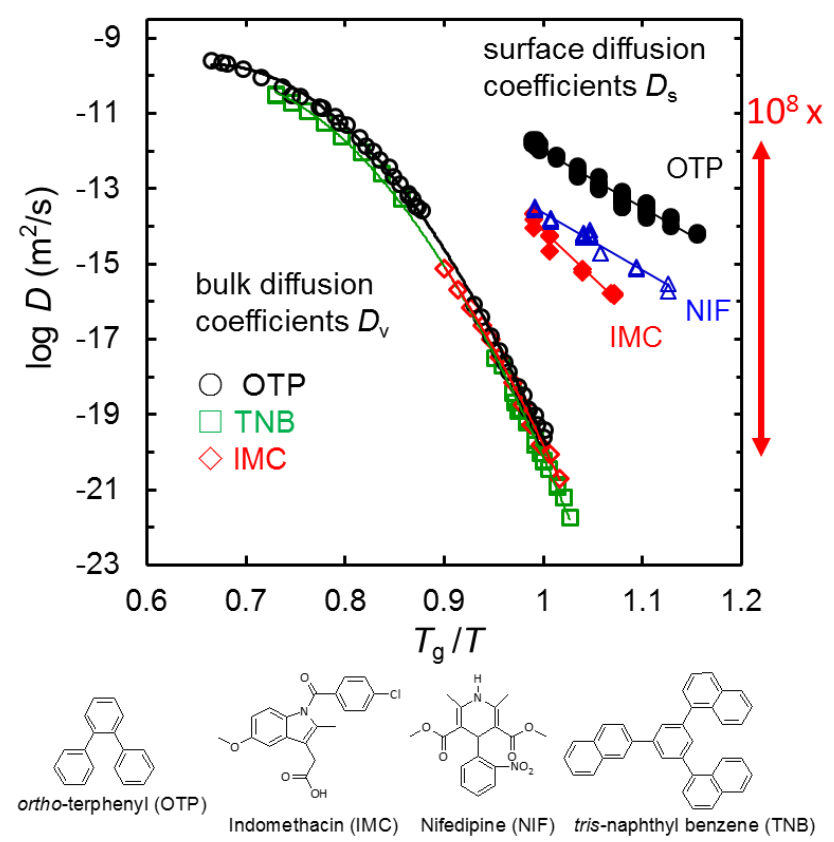
Figure 2. Surface and bulk diffusion coefficients $\left(D_{s}\right.$ and $D_{v}$ ) vs. $T_{\mathrm{g}} / T$. Note that $D_{\mathrm{s}}$ can be $10^{8}$ times larger than $D_{v}$ at $T_{\mathrm{g}}$. The $D_{v}$ values are clustered while the $D_{s}$ values show stronger molecular dependence.

$T_{\mathrm{g}}$, and has a higher activation energy. This effect is attributed to a steep mobility gradient beneath the surface and deeper penetration of polymer chains into the bulk. The molecular dependence of surface mobility suggests that surface-facilitated processes have system-dependent rates.

The surface diffusion coefficients in Figure 2 were obtained by the method of surface-grating decay. ${ }^{21}$ For molecular glasses, a sinusoidal grating pattern was embossed onto a free surface and its flattening was observed over time under the driving force of surface tension. ${ }^{12,13,14}$ While the flattening can occur by several mechanisms, surface diffusion dominates at short wavelengths. Mullins showed how the wavelength dependence of the decay rate $K$ can be used to determine the mechanism of surface evolution; ${ }^{21}$ for example, $K \propto \lambda^{-4}$ is the signature of surface diffusion and $K \propto \lambda^{-1}$ is the signature of viscous flow (collective motion of a liquid in a pressure gradient). While Mullins's method to measure surface diffusion was first applied to crystalline metals and silicon, simulations have shown that the method yields accurate surface diffusivity for glasses. ${ }^{22}$ 


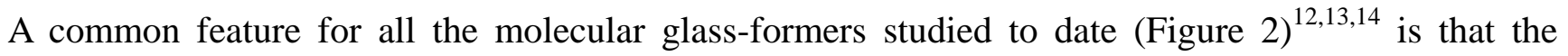
mechanism of surface evolution changes from viscous flow at high temperatures to surface diffusion at low temperatures. The viscosity at which this transition occurs $\eta_{t}$ depends on the rate of surface diffusion: slower surface diffusion allows viscous flow to control surface evolution up to a higher viscosity. Thus, $\eta_{\mathrm{t}}$ can be used to compare the surface diffusivity of different materials. For example, at $\lambda=1000 \mathrm{~nm}, \eta_{\mathrm{t}}=10^{6}-10^{8} \mathrm{~Pa} \mathrm{~s}$ for the small-molecule glasses in Figure 2, and $>10^{10} \mathrm{~Pa} \mathrm{~s}$ for polystyrenes, consistent with the latter's slower surface diffusion. ${ }^{14}$

Computer simulations have observed faster molecular rotation at the surface of a free-standing OTP film ${ }^{23}$ and faster diffusion at the surface of a glass-forming binary Lennard-Jones liquid. ${ }^{22,24}$ At present, the lowest temperature accessible to simulations is still far above the laboratory $T_{\mathrm{g}}$. For the binary LJ system, surface diffusion is $10^{3}$ times faster than bulk diffusion, $D_{\mathrm{s}} / D_{\mathrm{v}}=10^{3}$, when $D_{\mathrm{v}}=10^{-13} \mathrm{~m}^{2} / \mathrm{s}^{22}$ Similar result was obtained for the metallic glass $\mathrm{ZrNi}^{25}$ This enhancement ratio is consistent with the ratio for OTP and IMC, if we extrapolate the data in Figure 2 to high temperatures. This agreement suggests that the LJ model could capture the essential feature of surface mobility for van der Waals and metallic systems. For the LJ system, the transition from surface mobility to bulk mobility takes place over a distance of several particle diameters. ${ }^{22,24}$

Two equations have been proposed to predict the degree to which mobility is enhanced at a glass surface: ${ }^{26,27}$

$$
\begin{aligned}
& \tau_{\alpha} / \tau_{\text {surf }}=\left(\tau_{\alpha} / \tau_{0}\right)^{0.5} \\
& \tau_{\alpha} / \tau_{\text {surf }}=\left(\tau_{\alpha} / t_{\mathrm{c}}\right)^{n}
\end{aligned}
$$

In these equations, $\tau_{\alpha}$ and $\tau_{\text {surf }}$ are the bulk and surface relaxation times respectively. Eq. 1 originates from the Random First Order Transition (RFOT) theory, with $\tau_{0} \approx 1 \mathrm{ps},{ }^{26}$ and eq. 2 from the Coupling Model $(\mathrm{CM})$, where $t_{\mathrm{c}} \approx 2$ ps and $n$ is obtained from the Kohlrausch-Williams-Watt (KWW) exponent for fitting the bulk relaxation kinetics: $\Phi=\exp \left[-\left(t / \tau_{\alpha}\right)^{1-\mathrm{n}}\right] .{ }^{27}$ Both equations predict that surface mobility 
can be vastly higher than bulk mobility and have a weaker temperature dependence - features that also characterize surface diffusion on molecular glasses (Figure 2). A key difference between the two models is that eq. 1 predicts a universal relation between $\tau_{\alpha} / \tau_{\text {surf }}$ and $\tau_{\alpha}$, whereas eq. 2 predicts a materialdependent one.

Mirigian and Schweizer constructed a force-level statistical-mechanical model for structural relaxation near a free surface. ${ }^{28}$ The model is calibrated on the properties of actual glass-forming systems (e.g., OTP and polystyrene) and treats the faster dynamics of surface molecules as a result of fewer nearest neighbors and lower elastic cost for rearrangement. The model predicts a steep mobility gradient beneath the free surface, with the structural relaxation slowing by roughly a factor of $10^{6}$ per nm for polystyrene at $T_{\mathrm{g}}$. On the assumption that a molecule penetrates a depth on the order of its size and experiences a total friction that is the average of local frictions, the model predicts enhanced surface diffusion in approximate agreement with experiments (Figure 2), as well as the observed slowdown of surface diffusion with molecular size. ${ }^{12,13,14,20}$ 


\section{SURFACE MOBILITY AND ITS ROLE IN THE STABILITY OF MOLECULAR GLASSES}

If surface mobility is high, any process that requires molecular transport should be accelerated by a free surface. In this section, we consider the role of surface mobility in the crystallization of molecular glasses. Surface diffusion is directly linked to fast crystal growth at the free surface and may be partially responsible for fast crystal growth in the interior. We also consider the formation of stable glasses by vapor deposition as another consequence of surface mobility and surface-enhanced chemical degradation.

\section{Surface Mobility and Surface Crystallization. Recent}

work has found that molecular glasses can grow crystals much more rapidly at the free surface than in the interior. ${ }^{29,30,31,32,33,34}$ Here the phenomenon refers to the growth of crystals, not the nucleation (even though nucleation often occurs on free surfaces as well). The phenomenon has been observed for all the systems known to have fast surface diffusion - IMC, ${ }^{29} \mathrm{NIF},{ }^{30}$ and $\mathrm{OTP}^{34}$

(Figure 2), as well as systems whose surface diffusion has not been measured - griseofulvin, ${ }^{31}$ carbamazepine, ${ }^{32}$ and felodipine. ${ }^{33}$ To study this phenomenon, molecular glasses are prepared that do or do not have exposed free surfaces and crystal growth is observed in the two types of samples. Figure 3a shows the crystals growing on the free surface of an IMC glass. ${ }^{29,35,36}$ Surface crystals rise above the glass surface by tens to hundreds of nanometers as they grow

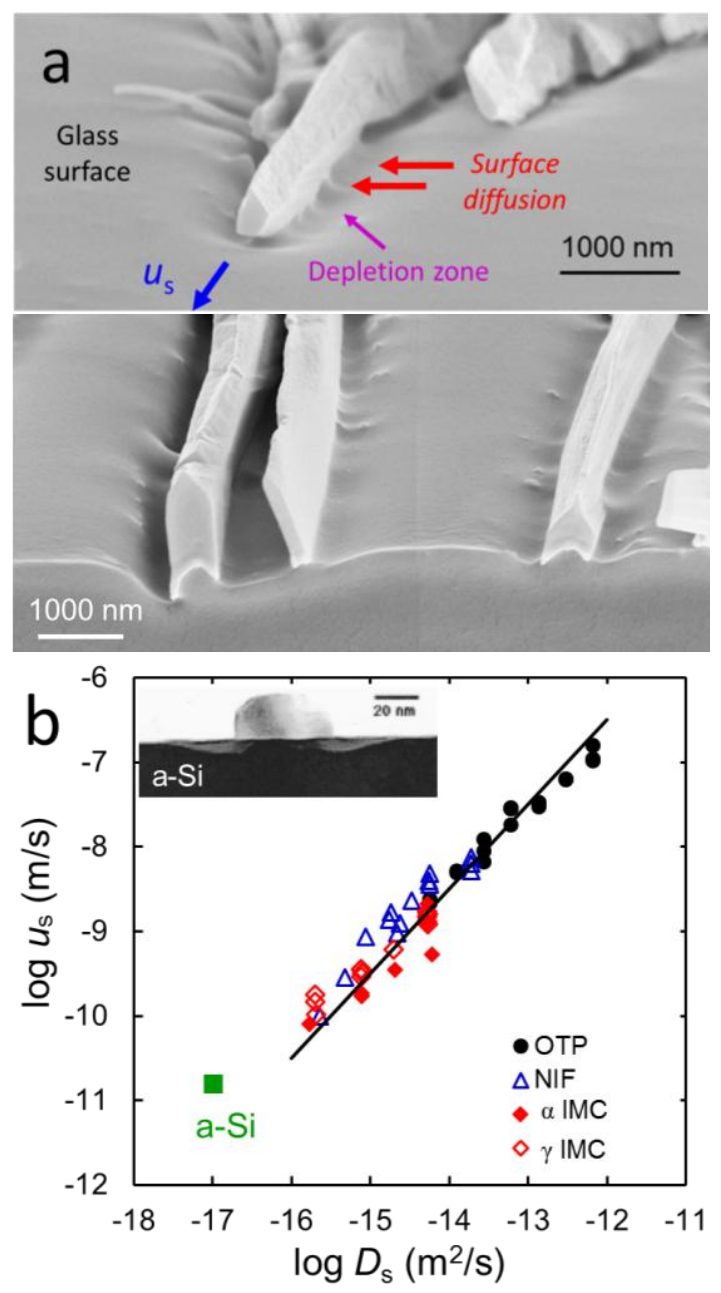

Figure 3. (a) Surface crystals on IMC glasses. (b) Velocity of surface crystal growth vs. surface diffusivity for 3 molecular glasses and amorphous silicon (a-Si). Inset shows a surface crystal on a-Si. ${ }^{39}$ 
laterally ( $u_{\mathrm{s}}$ indicates the lateral growth velocity). Meanwhile, they grow more slowly into the bulk. The fast surface crystal growth can be halted by a surface coating, even one a few $\mathrm{nm}$ thin, ${ }^{37}$ suggesting a key role for surface mobility in the process.

It is significant that the velocity of surface crystal growth $u_{\mathrm{s}}$ is approximately proportional to the coefficient of surface diffusion $D_{\mathrm{s}}$ (Figure $3 \mathrm{~b}$ ). This relation is observed for 3 molecular glasses (with IMC crystallizing in two polymorphs, $\alpha$ and $\gamma$ ) and one inorganic system (amorphous silicon ${ }^{38,39}$ ). This finding strongly indicates that surface mobility supports fast surface crystal growth. We envision that molecules diffuse on the glass surface to join the crystals (Figure 3a). Despite low bulk mobility, the glass manages to crystallize. Given that crystallization causes the failure of amorphous materials, establishing a link between surface mobility and crystallization is highly important.

Surface diffusion successfully explains a key feature of surface crystal growth: depletion zones around the crystals (Figure 3a). This feature results from a combined action of surface diffusion and crystallization flux. ${ }^{36}$ Near a slow-advancing front, the depletion zone widens and deepens over time in proportion to $t^{3 / 4}$ and $t^{1 / 4}$, respectively, precisely the kinetics expected for surface diffusion in the presence of a crystallization flux and not expected for other transport mechanisms. From independently measured surface diffusivity, ${ }^{12}$ Hasebe et al. predicted the evolution of depletion zones that agrees with experiment. This agreement strengthens the view that surface diffusion enables surface crystal growth.

Another feature of surface crystal growth on molecular glasses is its disruption by the onset of fluidity. ${ }^{34}$ Below $T_{\mathrm{g}}$, surface crystals grow at a steady rate; above $T_{\mathrm{g}}$, however, the process generally slows down and becomes unstable. This effect arises because liquid flow disrupts the manner in which crystals grow on the surface of a molecular glass (Figure 3): in the steady state, surface crystals draw molecules from the amorphous surface to grow upward and laterally; this process is possible only on a "solid ground", since the tendency of a flowing liquid is to wet and embed its own crystals. This effect indicates that surface crystal growth is a solid-state phenomenon deactivated in the liquid state. The 
observed response of surface crystal growth to the onset of fluidity is analogous to the response of the surface-grating decay noted earlier; in both cases, the mechanism of mass transport is surface diffusion at low temperatures and viscous flow at high temperatures. The two situations differ in that surface crystal growth slows down at the onset of liquid flow but the decay of surface gratings speeds up.

The different rates of surface and bulk crystallization can lead to complex crystallization kinetics. ${ }^{29,40,41}$ During isothermal storage of amorphous IMC, the degree of crystallinity rises initially and then approximately plateaus below $100 \%$ crystallinity; the plateau is higher if the particle size is smaller. In this two-stage crystallization, the initial rise of crystallinity corresponds to surface crystallization; after the free surface is fully covered, crystallization rate slows because the fast surface process is replaced by the slower bulk process. Smaller particles have larger specific surface area and reach higher degree of crystallinity at the end of surface crystallization. Besides IMC, this two-stage crystallization has been reported for amorphous griseofulvin, ${ }^{31}$ felodipine, ${ }^{40}$ and acetaminophen. ${ }^{41}$

Surface Mobility and Fast Bulk Crystallization through Fracture. Small-molecule glasses are brittle and easily fractured. ${ }^{42}$ Fracture creates free surfaces and is expected to accelerate crystallization. Consistent with this expectation, fractured molecular glasses have been observed to crystallize at a lower temperature upon heating. ${ }^{43,44,45}$ These observations suggest accelerated crystal nucleation by fracture surfaces. Powell et al. reported rapid crystal growth in OTP glasses with internal fracture created by tension from confining surfaces (Figure 4). ${ }^{46}$ Crystals grow rapidly along cracks, at a rate $u_{\mathrm{f}}$ roughly matching that on a free surface $u_{\mathrm{s}}$ and 10 times faster than the rate in the unfractured bulk $u_{\mathrm{b}}$. This result shows that fracture enables surface mobility to

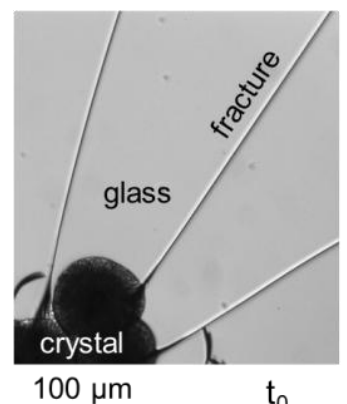

$100 \mu \mathrm{m}$

Figure 4. Fast crystal growth along internal cracks in an OTP glass at $T_{\mathrm{g}}-13 \mathrm{~K}^{46}$ accelerate local crystallization. Amorphous pharmaceutical solids can be fractured as a result of milling, 
compression, and impact. Given the brittleness of molecular glasses, fracture and surface mobility are a particularly serious threat to their stability.

Even in the absence of macroscopic fractures, surface mobility can still play an important role the bulk crystallization of molecular glasses. To see this connection, we briefly review the surprisingly fast crystal growth in the bulk of molecular glasses.

Figure 5 illustrates the phenomenon for OTP. Above $T_{\mathrm{g}}$, the velocity $u$ of crystal growth tracks the selfdiffusion coefficient $D$ over 5 orders of magnitude of change. ${ }^{47,48}$ This relation is expected by standard models that assume that bulk diffusion limits the rate of crystal growth. ${ }^{49}$ (The separation between $u$ and $D$ at higher temperatures reflects the decreasing thermodynamic driving force for crystallization as the system approaches the crystal melting point.) Extrapolating the diffusion-limited kinetics to low temperatures, one would expect very slow crystal

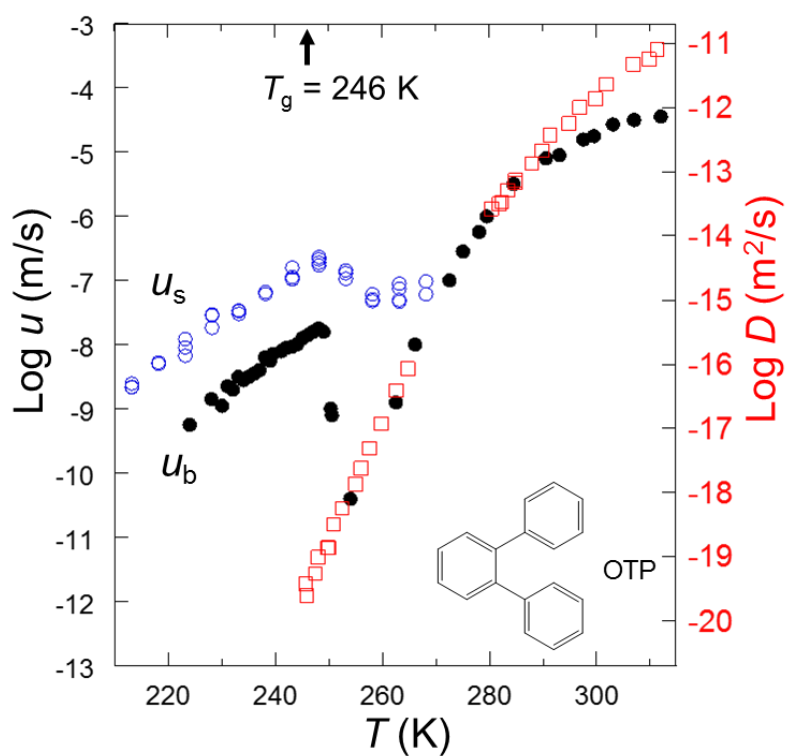

Figure 5. Crystal growth velocities in amorphous 0 terphenyl (OTP) on a free surface $u_{s}$ and in the bulk $u_{\mathrm{b}}$. The bulk growth is diffusion-limited at high temperatures and escapes this control at low temperatures (GC mode). The second y-axis shows bulk diffusivity. Reproduced from Ref. 46.

growth. This prediction proves to be grossly inaccurate: the actual velocities of growth are orders of magnitude faster than expected. ${ }^{50,51}$ This so-called glass-crystal or GC growth mode was first noted in 1967 by Greet and Turnbul1 ${ }^{50}$ and then studied systematically by Oguni and coworkers since $1995 .{ }^{51}$ The phenomenon is now known for many organic liquids. ${ }^{52,53}$

There have been many explanations for GC growth. ${ }^{46,51,54,55,56,57}$ These explanations envision different modes of molecular assembly and different roles for crystallization-induced stress (a result of growing denser crystals from a glass of lower density). In the most recent proposal, Powell et al. connect GC growth with fast surface crystal growth discussed earlier. ${ }^{46}$ For OTP, surface crystal growth is 10 times 
faster than bulk GC growth and has similar temperature dependence (Figure 5); both processes are active in the glassy state but disrupted above $T_{\mathrm{g}}$. These similarities suggest an underlying connection between the two processes and the possibility to understand GC growth through the better understood surface process. Powell et al. further rely on two experimental observations of GC growth: (1) it conserves the overall volume while creating denser crystalline domains ${ }^{46}$ and (2) it leads to a tension much smaller than expected for a strain equal to the crystal-glass density difference. ${ }^{58}$ These properties imply that GC growth continuously produces voids and free surfaces. Powell et al. propose that the free surfaces produced at the growth front accelerate local crystal growth. ${ }^{46}$ This idea has support from the fact that crystal growth is faster along existing cracks in a glass (Figure 4). This idea, if successful, would provide a single model that explains crystal growth both on the surface and in the interior of molecular glasses.

\section{Surface Mobility and Formation of Stable Glasses}

by Vapor Deposition. A recent discovery in glass science is that under suitable conditions, vapor deposition can prepare glasses of extraordinarily high density, low energy, and kinetic stability against thermal transformations. ${ }^{59,60}$ Here "suitable conditions" refer to a substrate temperature of roughly $0.85 T_{\mathrm{g}}$ and slow deposition rates. As shown in Figure $5,{ }^{60}$ a vapordeposited IMC glass transforms to the supercooled liquid at higher temperature with a greater input of energy than an ordinary liquid-cooled glass. While glass

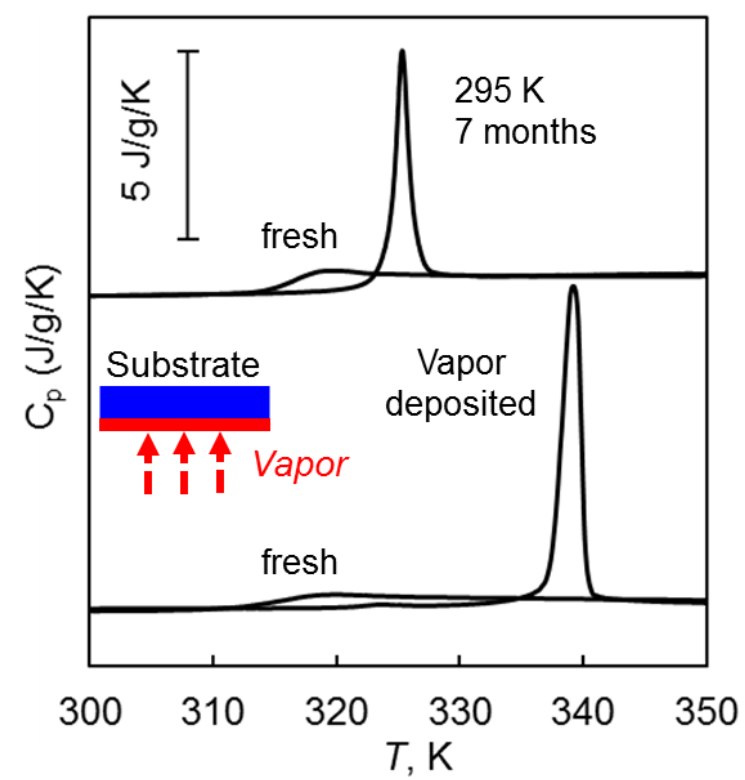

Figure 6. (a) Heat capacity of IMC glasses prepared by liquid-cooling and after aging. (b) Heat capacity of IMC glasses prepared by liquidcooling and vapor deposition. 
aging leads to changes in the same direction, very long aging is necessary to reach the same stability. For this material, aging for 7 months is not enough, ${ }^{61}$ and the required aging time would need to be one million years! The formation of stable glasses by vapor deposition has been observed for many materials, including many drugs. ${ }^{60}$

Surface mobility is responsible for the formation of stable glasses by vapor deposition. Surface mobility allows newly deposited molecules to equilibrate rapidly and organize into low-energy, highdensity structures before being buried by later arriving molecules. Repeating the process layer-by-layer produces a stable solid. It is noteworthy that stable glasses have been produced for all systems known to have fast surface diffusion: IMC, NIF, and OTP. ${ }^{59,60,62}$ Among these, the fastest surface diffuser OTP makes the most stable glass as measured by resistance to thermal transformation. ${ }^{62}$

Although glasses are usually isotropic, vapor-deposited molecular glasses can be anisotropic, with the molecules having preferential orientations with respect to the substrate. ${ }^{63,64,65}$ This phenomenon results from the anisotropic environment that molecules find themselves at the glass/vacuum interface during deposition and the ability for the deposition process to preserve and lock-in the orientational order in the product. The ability to control molecular orientation is potentially useful for organic electronics, while its pharmaceutical relevance is less clear.

Surface-Enhanced Chemical Degradation. Although this review is concerned mainly with physical transformations aided by surface mobility, it is relevant to mention examples of surface-facilitated chemical transformations in amorphous drug formulations. Xu et al. studied the degradation of amorphous protein formulations during storage in the solid state and found that "The majority of the degradation occurs in the population of protein molecules found on the solid surface." ${ }^{96}$ They further attributed the faster protein degradation at the surface to high surface mobility. Consistent with Xu et al., Constantino et al. observed that "spray freeze dried" proteins suffer more extensive degradation if the 
formulation has higher specific surface area. ${ }^{67}$ They proposed that "protein molecules exposed to the solid surface are more prone to deterioration". These studies suggest an important role for free surfaces in the chemical stability of amorphous pharmaceutical formulations. It is worth noting that the protein formulations discussed above have multiple components and the free surface could have a different chemical composition from the interior. ${ }^{68}$ Thus, surface-enhanced degradation could be a result of surface mobility, depletion of stabilizing excipients in the surface region, or both.

Stabilization against Surface-Facilitated Transformations. Given that free surfaces can speed up physicochemical transformations, one way to improve the stability of molecular glasses is to reduce the specific surface area. For systems showing fast surface crystallization, increasing the particle size can slow the rate of overall crystallization (because the surface process affects a smaller fraction of the material). ${ }^{29,31,40,41}$ A second way to prevent surface-facilitated transformations is coating the free surface. Under a coating, surface crystal growth on molecular glasses can be inhibited. ${ }^{30,31,37}$ It is noteworthy that even an extremely thin can be effective for this purpose; for example, a polymer layer only a few nm thin. ${ }^{37}$ The very thin coating makes it possible to improve stability with the addition of only a small amount of polymers. Besides solution-applied coatings, dry coatings can be effective for inhibiting surface crystallization. ${ }^{41,69}$ Loading amorphous drugs in porous inorganic glasses can improve stability ${ }^{70}$ and the effect may involve the reduction of free surfaces and the contact with inert solid surfaces.

Given that surface crystals growing on molecular glasses have characteristic dimensions or "footprints", it might be possible to disrupt their growth by reducing the space available. The typical footprint for surface crystals is on the order of one micrometer (the size of the crystal plus the size of the depletion zones; see Figure 3) ${ }^{36}$ Limiting or dividing the space accessible to this dimension could frustrate surface crystal growth. 
Fracture creates free surfaces and preventing fracture may improve stability. Recent work has shown that while small-molecule glasses are easy to fracture, polymer additives can effectively increase their fracture resistance. ${ }^{42}$ For example, $10 \mathrm{wt} \%$ polystyrene $\left(M_{\mathrm{w}}=1 \mathrm{million} \mathrm{g} / \mathrm{mole}\right)$ increases the critical energy release rate of OTP from 0.9 to $4.5 \mathrm{~J} / \mathrm{m}^{2}$. This effect can be explained by the fracture tip having to go around the volume pervaded by each polymer chain encountered, increasing the energetic cost of fracture. This effect could be partially responsible for the strong inhibitory effect of certain polymers on crystal growth in the interior of molecular glasses, even at low concentrations, despite their weaker

effect on surface crystal growth. ${ }^{71}$ Combining bulk doping and surface coating may be an efficient way to produce physically stable amorphous drugs that contain only a small amount of polymers.

\section{CONCLUDING REMARKS}

Recent work has found that surface mobility can be extremely high on molecular glasses and play a key role in their physical stability. Surface mobility is directly linked to fast surface crystal growth and may be involved in bulk crystal growth through the creation of voids and free surfaces. Surface mobility is responsible for the formation of stable glasses by vapor deposition; in terms of density and energy, these glasses are equivalent to liquid-cooled glasses that have been aged for thousands of years. There is evidence that free surfaces can accelerate the chemical degradation of protein formations, though it is unclear whether the effect arises from high surface mobility, surface depletion of stabilizers, or both. These findings indicate that bulk properties alone are insufficient for understanding the stability of amorphous drugs and that an efficient way to improve stability may be the inhibition of surface-aided transformations.

The important role of surface mobility in glass science motivates a fundamental understanding of this property in developing stable amorphous materials. One of the open questions concerns the molecular dependence of surface mobility. At the same bulk diffusivity, surface diffusivity can be significantly 
different (Figure 2), apparently slowing down with increasing molecular size and intermolecular forces; this trend seems to continue from molecular glasses to the glasses of polystyrenes and silicates, with the latter having stronger intermolecular forces and slower surface diffusion. Future work is needed for a fuller understanding of this dependence. Given that many important amorphous materials contain multiple components (e.g., drug-polymer solid dispersions), it is relevant to understand surface transport in these systems; for example, whether the simultaneous diffusion of fast and slow species leads to surface segregation of components. Here progress can be expected by combining surface dynamics measurements with high-resolution three-dimensional mapping of chemical components.

ACKNOWLEDGMENT. I thank the National Science Foundation (DMR 1206724 and 1234320) for supporting this work and my coworkers for their contributions, including M. D. Ediger, Juan J. de Pablo, Lei Zhu, Ye Sun, Hanmi Xi, Caleb Brian, Wei Zhang, Tian Wu, Erica Gunn, Ting Cai, Mariko Hasebe, Danielle Musumeci, C. Travis Powell, and Yinshan Chen.

\section{REFERENCES}

${ }^{1}$ Chen, M. Mechanical Behavior of Metallic Glasses: Microscopic Understanding of Strength and Ductility. Annu. Rev. Mater. Res. 2008, 38, 445-469.

${ }^{2}$ Johnson, W. L.; Samwer, K. A Universal Criterion for Plastic Yielding of Metallic Glasses with a $\left(T / T_{\mathrm{g}}\right)^{2 / 3}$ Temperature Dependence. Phys. Rev. Lett. 2005, 95, 195501-1 to 195501-4.

${ }^{3}$ Hancock, B. C.; Zografi, G. Characteristics and Significance of the Amorphous State in Pharmaceutical Systems J. Pharm. Sci. 1997, 86, 1-12. 
${ }^{4} \mathrm{Yu}, \mathrm{L}$. Amorphous pharmaceutical solids: preparation, characterization and stabilization. Advanced Drug Delivery Reviews 2001, 48, 27-42.

${ }^{5}$ Bhugra, C.; Pikal M. J. Role of Thermodynamic, Molecular and Kinetic Factors in Crystallization from the Amorphous State J. Pharm. Sci. 2008, 97, 1329-1349.

${ }^{6}$ Fahy, G.M.; MacFarlane, D.R.; Angell, C.A.; Meryman, H.T. Vitrification as an approach to cryopreservation. Cryobiology 1984, 21, 407-426.

${ }^{7}$ Crowe, J. H.; Carpenter, J. F.; Crowe, L. M. The Role of Vitrification in Anhydrobiosis. Annu. Rev. Physiol. 1998, 60, 73-103.

${ }^{8}$ Shirota, Y. Photo- and electroactive amorphous molecular materials - molecular design, syntheses, reactions, properties, and applications. J. Mater. Chem. 2005, 15, 75-93.

${ }^{9}$ De Silva, A.; Felix, N. M.; Ober, C. K. Molecular Glass Resists as High-Resolution Patterning Materials. Adv. Mater. 2008, 20, 3355-3361.

${ }^{10}$ Sun, Y.; Zhu, L.; Wu, T.; Cai, T.; Gunn, E. M.; Yu, L. Stability of Amorphous Pharmaceutical Solids: Crystal Growth Mechanisms and Effect of Polymer Additives. AAPS Journal. 2012, 14, 380388.

11 Bhattacharya, S.; Suryanarayanan, R. Local mobility in amorphous pharmaceuticals characterization and implications on stability. J. Pharm. Sci. 2009, 98, 2935-2953.

${ }^{12}$ Zhu, L.; Brian, C.W.; Swallen, S.F.; Straus, P.T.; Ediger, M.D.; Yu, L. Surface Self-Diffusion of an Organic Glass. Phys. Rev. Lett. 2011, 106, 256103-1 - 256103-4. 
${ }^{13}$ Brian, C. W.; Yu, L. Surface Self-Diffusion of Organic Glasses. J. Phys. Chem. A 2013, 117, 13303 - 13309.

${ }^{14}$ Zhang, W.; Brian, C. W.; Yu, L. Fast Surface Diffusion of Amorphous $o$-Terphenyl and its Competition with Viscous Flow in Surface Evolution. J. Phys. Chem. B. 2015, 119, 5071-5078.

${ }^{15}$ Yang, Z.; Fujii, Y.; Lee, F. K.; Lam, C.; Tsui, O. K. C. Glass Transition Dynamics and Surface Layer Mobility in Unentangled Polystyrene Films. Science 2010, 328, 1676-1679.

${ }^{16}$ Chai, Y.; Salez, T.; McGraw, J. D.; Benzaquen, M.; Dalnoki-Veress, K.; Raphaël, E.; Forrest, J. A. A Direct Quantitative Measure of Surface Mobility in a Glassy Polymer. Science 2014, 343, 994-999.

${ }^{17}$ Bell, R.C.; Wang, H.F.; Iedema, M.J.; Cowin, J.P. Nanometer-Resolved Interfacial Fluidity. J. Am. Chem. Soc. 2003, 125, 5176-5185.

${ }^{18}$ Daley et al. Comparing surface and bulk flow of a molecular glass former. Soft Matter 2012, 8, 2206.

${ }^{19}$ Paeng, K.; Swallen, S. F.; Ediger, M. D. J. Am. Chem. Soc. 2011, 133 (22), 8444- 8447.

${ }^{20}$ Zhang, W.; Yu, L. Surface diffusion of polymer glasses. Macromolecules 2016, in press.

${ }^{21}$ Mullins, W. W. Flattening of a Nearly Plane Solid Surface due to Capillarity. J. Appl. Phys. 1959, $30,77-83$.

${ }^{22}$ Malshe, R.; Ediger, M. D.; Yu, L.; de Pablo, J. J. Evolution of glassy gratings with variable aspect ratios under surface diffusion. J. Chem. Phys. 2011, 134(19), 194704/1-194704/8. 
${ }^{23}$ Ghosh, J.; Faller, R. A comparative molecular simulation study of the glass former ortho-terphenyl in bulk and freestanding films. J. Chem. Phys. 2006, 125, 044506.

${ }^{24}$ Amir Haji-Akbari and Pablo G. Debenedetti, The effect of substrate on thermodynamic and kinetic anisotropies in atomic thin films. J. Chem. Phys. 141, 024506 (2014)

${ }^{25}$ Boddeker, B.; Teichler, H. Dynamics near free surfaces of molecular dynamics simulated $\mathrm{Ni}_{0.5} \mathrm{Zr}_{0.5}$ metallic glass films. Phys. Rev. B 1999, 59, 1948-1956.

${ }^{26}$ Stevenson, J. D.; Wolynes, P. G. On the Surface of Glasses. J. Chem. Phys. 2008, 129, 234514.

${ }^{27}$ Capaccioli, S.; Ngai, K. L.; Paluch, M.; Prevosto, D. Mechanism of Fast Surface Self-Diffusion of an Organic Glass. Phys. Rev. E 2012, 86, 051503.

${ }^{28}$ Mirigian, S.; Schweizer, K. S. Theory of activated glassy relaxation, mobility gradients, surface diffusion, and vitrification in free standing thin films. J. Chem. Phys. 2015, 143, 244705.

${ }^{29}$ Wu, T.; Yu, L. Surface Crystallization of Indomethacin Below Glass Transition Temperature Pharm. Res. 2006, 23, 2350-2355.

${ }^{30}$ Zhu, L.; Wong, L.; Yu, L. Surface-Enhanced Crystallization of Amorphous Nifedipine Mol. Pharm. 2008, 5, 921-926.

${ }^{31}$ Zhu, L.; Jona, J.; Nagapudi, K.; Wu, T. Fast Surface Crystallization of Amorphous Griseofulvin Below $T_{\mathrm{g} .}$ Pharm. Res. 2010, 27, 1558-1567.

${ }^{32}$ Gunn, E.; Guzei, I. A.; Yu, L. Does Crystal Density Control Fast Surface Crystal Growth in Glasses? A Study with Polymorphs. Cryst. Growth Des. 2011, 11(9), 3979-3984. 
${ }^{33}$ Kestur, U. S.; Taylor, L. S. Evaluation of the Crystal Growth Rate of Felodipine Polymorphs in the Presence and Absence of Additives As a Function of Temperature. Cryst. Growth Des. 2013, 13, 43494354.

${ }^{34}$ Hasebe, M.; Musumeci, D.; Powell, C. T.; Ting, C.; Gunn, E.; Zhu, L.; Yu, L. Fast Surface Crystal Growth on Molecular Glasses and Its Termination by the Onset of Fluidity. J. Phys. Chem. B. 2014, $118,7638-7646$

${ }^{35}$ Sun, Y.; Zhu, L.; Kearns, K. L.; Ediger, M. D.; Yu, L. Glasses Crystallize Rapidly at Free Surfaces by Growing Crystals Upward. Proc. Natl. Acad. Sci. 2011, 108, 5990-5995.

${ }^{36}$ Hasebe, M.; Musumeci, D.; Yu, L. Fast Surface Crystallization of Molecular Glasses: Creation of Depletion Zones by Surface Diffusion and Crystallization Flux. J. Phys. Chem. B. 2015, 119, 33043311.

${ }^{37}$ Wu, T.; Sun, Y.; Li, N.; De Villiers, M. M.; Yu, L. Inhibiting Surface Crystallization of Amorphous Indomethacin by Nanocoating. Langmuir 2007, 23, 5148-5153.

38 Sakai, A.; Tatsumi, T.; Ishida, K. Growth Kinetics of Si Hemispherical Grains on Clean Amorphous-Si Surfaces. J. Vacuum Sci. \& Tech. A: Vacuum, Surfaces, \& Films 1993, 11, 2950-2953.

${ }^{39}$ Sallese, J. M.; Ils, A.; Bouvet, D.; Fazan, P. Modeling of the Depletion of the Amorphous-Silicon Surface during Hemispherical Grained Silicon Formation. J. Appl. Phys. 2000, 88, 5751 - 5755.

${ }^{40}$ Kestur, U.; Ivanesivic, I.; Alonzo, D.; Taylor, L. Influence of particle size on the crystallization kinetics of amorphous felodipine powders. Powder Technol. 2013, 236, 197-204. 
${ }^{41}$ Capece, M.; Dave, R. Enhanced Physical Stability of Amorphous Drug Formulations via Dry Polymer Coating. J. Pharm. Sci. 2015, 104, 2076-2084.

${ }^{42}$ Powell, C. T.; Chen, Y.; Yu, L. Fracture of molecular glasses under tension and increasing their fracture resistance by polymer additives. J. Non-Crystalline Solids 2015, 429, 122-128.

${ }^{43}$ Legrand, V.; Descamps, M.; Alba-Simionesco, C. Thermochimica Acta 1997, 307, 77-83.

${ }^{44}$ Bhugra, C.; Shmeis, R.; Pikal, M. J. Role of Mechanical Stress in Crystallization and Relaxation Behavior of Amorphous Indomethacin. J. Pharm. Sci. 2008, 97, 4446 -4458.

45 Ayenew, Z.; Paudel, A.; Rombaut, P.; van den Mooter, G. Effect of Compression on Nonisothermal Crystallization Behaviour of Amorphous Indomethacin. Pharm. Res. 2012, 29, 2489-2498.

${ }^{46}$ Powell, C. T.; Xi, H.; Sun, Y.; Gunn, E.; Chen, Y.; Ediger, M. D.; Yu, L. Fast Crystal Growth in $o-$ Terphenyl Glasses: A Possible Role for Fracture and Surface Mobility. J. Phys. Chem. B 2015, 119, 10124-10130.

${ }^{47}$ Magill, J. H.; Li, H. M. Physical Properties of Aromatic Hydrocarbons. V. Solidification Behavior of 1,2-Diphenylbenzene. J. Crystal Growth 1973, 20, 135-44.

${ }^{48}$ Mapes, M. K.; Swallen, S. F.; Ediger, M. D. Self-Diffusion of Supercooled o-Terphenyl near the Glass Transition Temperature. J. Phys. Chem. B 2006, 110, 507-511.

${ }^{49}$ Ediger, M. D.; Harrowell, P.; Yu, L. Crystal Growth Kinetics Exhibit a Fragility-Dependent Decoupling from Viscosity. J. Chem. Phys. 2008, 128, 034709/1-034709/6.

${ }^{50}$ R. J. Greet, D. J. Turnbull, Glass Transition in $o$-Terphenyl. J. Chem. Phys. 1967, 46, 1243-1251. 
${ }^{51}$ Hikima, T.; Adachi, Y.; Hanaya, M.; Oguni, M. Determination of Potentially HomogeneousNucleation-Based Crystallization in $o$-Terphenyl and an Interpretation of the Nucleation-Enhancement Mechanism. Phys. Rev. B 1995, 52, 3900-3908.

${ }^{52}$ Hatase, M.; Hanaya, M.; Oguni, M. Studies of Homogeneous-Nucleation-Based Crystal Growth: Significant Role of Phenyl Ring in the Structure Formation. J. Non-Cryst. Solids 2004, 333, 129-136.

${ }^{53}$ Musumeci, D.; Powell, C. T.; Ediger, M. D.; Yu, L. Termination of Solid-State Crystal Growth in Molecular Glasses by Fluidity. J. Phys. Chem. Lett. 2014, 5, 1705-1710.

${ }^{54}$ Sun, Y.; Xi, H.; Chen, S.; Ediger, M. D.; Yu, L. Crystallization near Glass Transition: Transition from Diffusion-Controlled to Diffusionless Crystal Growth Studied with Seven Polymorphs. J. Phys. Chem. B 2008, 112, 5594-5601.

${ }^{55}$ Konishi, T.; Tanaka, H. Possible Origin of Enhanced Crystal Growth in a Glass. Phys. Rev. B 2007, 76, 220201.

${ }^{56}$ Stevenson, J. D.; Wolynes, P. G. The Ultimate Fate of Supercooled Liquids. J. Phys. Chem. A 2011, $115,3713-3719$.

${ }^{57}$ Caroli, C.; Lemaître, A. Ultrafast Spherulitic Crystal Growth as a Stress-Induced Phenomenon Specific of Fragile Glass-Formers. J. Chem. Phys. 2012, 137, 114506-1 to 114506-9.

${ }^{58}$ Paeng, K.; Powell, C. T.; Yu, L.; Ediger, M. D. Fast crystal growth induces mobility and tension in supercooled $o$-terphenyl. J. Phys. Chem. Lett. 2012, 3, 2562-2567.

${ }^{59}$ Swallen, S.; Kearns, K.; Mapes, M.; McMahon, R.; Kim, S.; Ediger, M.; Yu, L.; Wu, T.; Satija, S. Extraordinarily Stable Glassy Materials Prepared by Vapor Deposition. Science 2007, 315, 353 - 356. 
${ }^{60}$ Zhu, L.; Yu, L. Generality of forming stable organic glasses by vapor deposition. Chem. Phys. Lett. 2011, 499, 62-65.

${ }^{61}$ Brian, C. W.; Zhu, L.; Yu, L. Effect of Bulk Aging on Surface Diffusion of Organic Glasses. J. Chem. Phys. 2014, 140, 054509/1-6.

${ }^{62}$ Whitaker, K. R.; Tylinski, M.; Ahrenberg, M.; Schick, C.; Ediger, M. D. Kinetic stability and heat capacity of vapor-deposited glasses of $o$-terphenyl. J. Chem. Phys. 2015, 143, 084511-1 to 084511-10.

${ }^{63}$ Yokoyama, D.; Setoguchi, Y.; Sakaguchi, A.; Suzuki, M.; Adachi, C. Orientation Control of LinearShaped Molecules in Vacuum-Deposited Organic Amorphous Films and Its Effect on Carrier Mobilities. Adv Funct Mater 2010, 20(3), 386-391.

${ }^{64}$ Dawson, K. J.; Zhu, L.; Yu, L.; Ediger, M. D. Anisotropic structure and transformation kinetics of vapor-deposited indomethacin glasses. J. Phys. Chem. B. 2011, 115, 455-463.

${ }^{65}$ Dalal, S., et al. Tunable molecular orientation and elevated thermal stability of vapor-deposited organic semiconductors. Proc. Natl. Acad. Sci. 2015, 112, 14.

${ }^{66}$ Xu, Y.; Grobelny, P.; von Allmen, A.; Knudson, K.; Pikal, M.; Carpenter, J. F.; Randolph, T. W. Protein Quantity on the Air-Solid Interface Determines Degradation Rates of Human Growth Hormone in Lyophilized Samples. J. Pharm. Sci. 2014, 103, 1356-1366.

${ }^{67}$ Constantino et al. J. Pharm. Sci. 2002, 91, 388-395.

${ }^{68}$ Abdul-Fattah, A. M.; Lechuga-Ballesteros, D.; Kalonia, D. S.; Pikal, M. J. The Impact of Drying Method and Formulation on the Physical Properties and Stability of Methionyl Human Growth Hormone in the Amorphous Solid State. J. Pharm. Sci. 2008, 97, 163 - 184. 
${ }^{69}$ Priemel, P. A.; Laitinen, R.; Barthold, S.; Grohganz, H.; Lehto, V.; Rades, R.; Strachan, C. J. Inhibition of surface crystallisation of amorphous indomethacin particles in physical drug-polymer mixtures. Int. J. Pharm. 2013, 456, 301.

${ }^{70}$ Qian, K. K.; Bogner, R. H. Application of Mesoporous Silicon Dioxide and Silicate in Oral Amorphous Drug Delivery Systems. J. Pham. Sci. 2012, 101, 444-463.

${ }^{71}$ Powell, C. T.; Cai, T.; Hasebe, M.; Gunn, E. M.; Gao, P.; Zhang, G.; Gong, Y.; Yu, L. LowConcentration Polymers Inhibit and Accelerate Crystal Growth in Organic Glasses in Correlation with Segmental Mobility. J. Phys. Chem. B 2013, 117, 10334-10341. 